\title{
A Rare Case of Nocturnal Urinary Incontinence and Menuria after Lower Segment Cesarean Section
}

\author{
${ }^{1}$ Priyakshi Chaudhry, ${ }^{2}$ Deepti S Shrivastava, ${ }^{3}$ Sindhu Bhute, ${ }^{4}$ Suhas Jajoo
}

\begin{abstract}
Misgav Ladach technique for lower segment cesarean section (LSCS) is considered advantageous universally, but debate still continues about nonclosure in layers and further consequences; hence, careful selection of cases and intraoperative decision of closure in layers is important. The classical Youssef's syndrome comprises cyclic hematuria, amenorrhea, menuria, and complete urinary continence in a patient who had LSCS. Hereby, we present a case report of a woman who suffered with chronic pelvic pain, menuria, and nocturnal eneuresis after her LSCS for obstructed labor, although not exactly the same as Youssef syndrome but rare in occurrence and relieved after adhesiolysis and gonadotropin-releasing hormone $(\mathrm{GnRH})$ analog therapy.
\end{abstract}

Keywords: Menuria, Nocturnal eneuresis, Obstructed labor.

How to cite this article: Chaudhry P, Shrivastava DS, Bhute S, Jajoo S. A Rare Case of Nocturnal Urinary Incontinence and Menuria after Lower Segment Cesarean Section. J Med Sci 2016;2(1):23-25.

Source of support: Nil

Conflict of interest: None

\section{INTRODUCTION}

Lower segment cesarean section (LSCS) by Misgav Ladach technique is the most frequently performed surgery by obstetricians today. ${ }^{1}$ Incidences of postoperative adhesion formation and urinary bladder endometriosis depend on individual immune response of patient and to some extent on the skill of the operator and careful selection of patients. Recently, peritoneal closure in two layers is documented by some studies to have lesser adhesion formation. ${ }^{2,3}$

Due to its fast and minimalist approach, it has been adopted by obstetricians worldwide, but sometimes dense adhesions are presented as a complication afterward. ${ }^{4}$ We report one such case of overflow incontinence and menuria due to dense adhesions and bladder wall endometriosis following primary LSCS.

\footnotetext{
${ }^{1}$ Resident, ${ }^{2}$ Professor and Head, ${ }^{3,4}$ Professor

${ }^{1-4}$ Department of Obstetrics and Gynecology, Jawaharlal Nehru Medical College, Sawangi, Maharashtra, India

Corresponding Author: Priyakshi Chaudhry, Resident Department of Obstetrics and Gynecology, Jawaharlal Nehru Medical College, Sawangi, Maharashtra, India, Phone: +919975459078, e-mail: priyakshichaudhry@gmail.com
}

\section{CASE REPORT}

A 23-year-old $\mathrm{P}_{1} \mathrm{~L}_{1}$ was admitted with chronic pelvic pain, nocturnal bed wetting, and cyclic hematuria during periods following her last cesarean section, performed 2 years earlier in view of prolonged second stage of labor. In day time, she had frequency of urine but denied any kind of incontinence. She was kept catheterized for 45 days postoperatively due to hematuria at the time of LSCS. She noticed menuria too, when she resumed her cycles after the LSCS. Her menstrual flow through the genital tract was average in volume.

Her general and systemic examination findings were normal. She had a transverse cesarean scar. Pelvic examination revealed a normal-sized anteverted uterus with restricted mobility and induration felt through fornices. Ultrasonography revealed normal-sized uterus and normal urinary bladder and adnexae. Diagnostic hysterolaparoscopy with cystoscopy was planned. On cystoscopy, a small depression in posterior bladder wall was seen covered with clots; methylene blue was instilled through cervix to see any spillage from bladder wall, but it was negative. From suspicious tissue, biopsy was sent for histopathological examination. On laparoscopy, anterior surface of uterus was seen completely adherent to anterior abdominal wall, and only upper surface of uterus was visualized surrounded by adhesions all over (Fig. 1).

Laparotomy was done to rule out any vesico-uterine fistula (VUF) and dissecting uterus. In the same sitting the

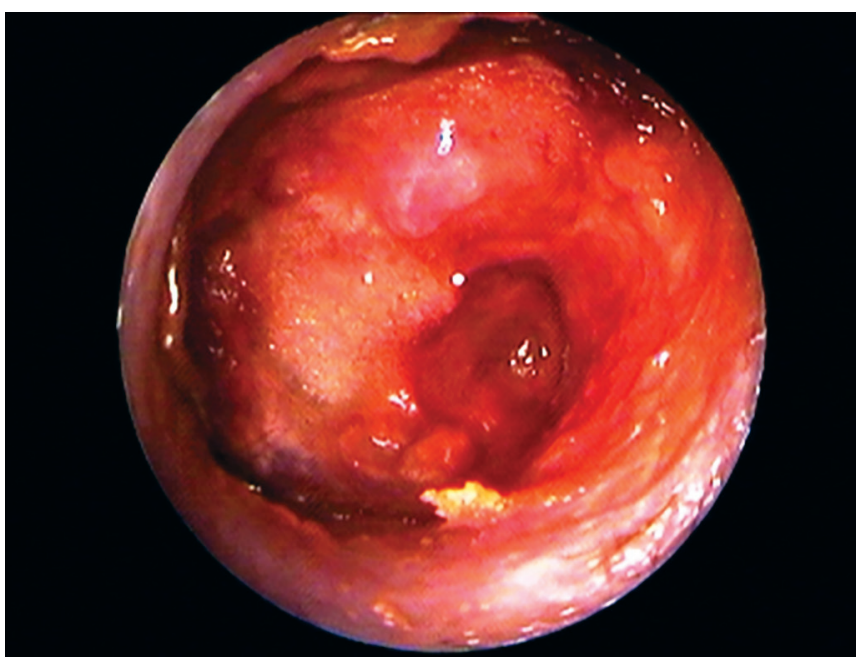

Fig. 1: Laparoscopy picture showing only upper surface of uterus visualized surrounded all over by adhesions 


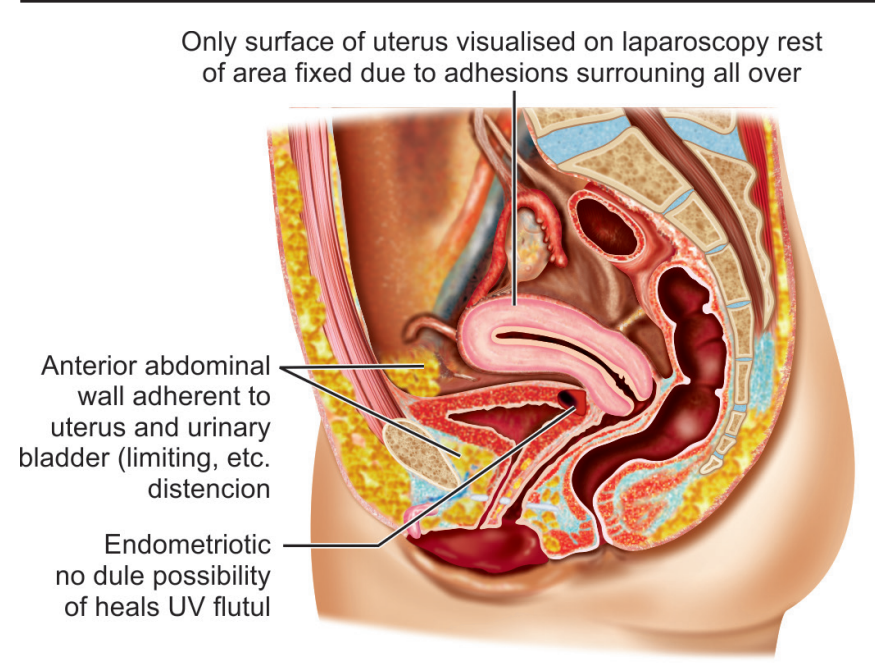

Fig. 2: Laparotomy picture, i.e., uterus adherent all around limiting distension of urinary bladder. Endometriotic nodule visualized on cystoscopy

urinary bladder adhesions were released from the anterior abdominal wall. On laparotomy, after some adhesiolysis, uterus was appeared sitting over the urinary bladder and densely fixed to the anterior abdominal wall (Fig. 2). Even after complete separation of uterus and urinary bladder, no fistulous track was observed in between; there appeared initial injury during primary LSCS, which was healed leaving behind some endometrial tissue in the bladder wall. Omental pad was kept in between uterus and urinary bladder. Postoperative period was uneventful. Biopsy from urinary bladder confirmed endometrial glands. She was discharged on Injection leuprolide $1.25 \mathrm{mg}$ for 3 months.

Her urinary incontinence was totally resolved and when the patient resumed her cycles, there was no menuria.

\section{DISCUSSION}

Adhesion formation after cesarean section varies widely in incidence. Its etiopathology is poorly understood. It is again debatable whether peritoneal closure is beneficial or not. ${ }^{2-4}$ Endometriosis is defined as the presence of functional endometrial tissue outside the uterine cavity. 5,6 Endometriosis of the urinary bladder is an uncommon lesion and is seen in $1 \%$ of all cases of endometriosis. ${ }^{7}$ It exists in two forms, primary and secondary. The primary form is generally a part of generalized pelvic disease, whereas the secondary is iatrogenic, i.e., it occurs after pelvic surgery like cesarean section or hysterectomy. ${ }^{7}$ In this case, it could have developed following prolonged second stage of labor or due to intraoperative trauma leading to VUF, which healed afterward. Vesicouterine fistula is an uncommon urogenital fistula and accounts for 1 to $4 \%$ of all urogenital fistulas. ${ }^{8}$ Cyclical hematuria or menuria is an important clinical feature of this fistula, which may or may not be associated with urinary incontinence depending on the location of the fistulous tract. ${ }^{6}$ The classical Youssef syndrome comprises cyclic hematuria, amenorrhea, menuria, and complete urinary continence in a patient who had LSCS. ${ }^{9}$ This is explained by the differential pressure gradient between the uterus and the bladder and the sphincteric action of the isthmus, which facilitates passage of blood from the uterus into the bladder. ${ }^{9}$ Our case is not a classical Youssef's syndrome as the patient had incontinence. Dense adhesions between the anterior abdominal wall, uterus, and bladder were noted on laparoscopy and confirmed on laparotomy. The anchoring effect of the uterus on bladder explains the overflow incontinence experienced by the patient. Total separation of uterus, urinary bladder, and anterior abdominal wall by laparotomy relieved the urinary incontinence of the patient. The diagnosis of VUF is often confirmed by imaging studies and cystoscopy. ${ }^{7,9}$ Vesicouterine fistula following cesarean section may heal spontaneously with involution of the puerperal uterus. Spontaneous healing may occur in $5 \%$ of cases. When it does not, continuous hormonal therapy can be given to suppress menstruation for 3 to 6 months as first line of therapy. Suppression of menstruation can be tried with progestogens or gonadotropin-releasing hormone analogs, ${ }^{6}$ as is done in this case. The histopathology confirmed endometriosis of bladder wall.

\section{CONCLUSION}

Injuries to the bladder discovered at the time of cesarean section should be repaired immediately. If the diagnosis of a VUF is made in the early postoperative period, spontaneous closure of fistula with continuous urethral catheter drainage for 2 weeks with antibiotic cover occurs. ${ }^{8}$ Diagnosis of such cases are difficult due to nonspecific symptoms. High index of suspicion to all symptomatic women with a history of cesarean delivery or other gynecological surgery gives a clue to the diagnosis.

Ultrasonography may be inconclusive. Cystoscopy and biopsy may give a clue to the diagnosis before surgery. ${ }^{9}$ Treatment varies according to the severity and site of involvement of each case. Hormonal therapy does have a definite role in regressing the lesion. ${ }^{6}$

Although Misgav Ladach technique is advantageous in general for LSCS, care should be taken for proper selection of cases. Specially in cases of obstructed labor where bladder wall integrity is unpredictable, layer-wise opening and closure is important to prevent postoperative complications. 


\section{REFERENCES}

1. Shetty A, Fonseca M, Rao S, Badhwar VR. The Misgav Ladach lower segment caesarean section experience at a tertiary hospital. Bombay Hospital J. Available from: www.bhj.org/ journal/2003_4502_april/themisgav_294.htm.

2. Lyell DJ, Caughey AB, Hu E, Daniels K. Peritoneal closure at primary cesarean delivery and adhesions. Obstet Gynecol 2005 Aug;106(2):275-280.

3. Tulandi T, Al-Sunaidi M. Peritoneal closure at C-section reduces the risk of adhesions. J Fam Pract 2007 Sept;19 (9):9.

4. Shi Z, Ma L, Yang Y, Wang H, Schreiber A, Li X, Tai S, Zhao X, Teng J, Zhang L, et al. Adhesion formation after previous caesarean section-a meta-analysis and systematic review. BJOG 2011 Mar;118(4):410-422.
5. Khetan N, Torkington J, Watkint A, Jamison MH, Humphreys WV. Endometriosis: presentation to general surgeons. Ann R Coll Surg Engl 1999 Jul;81(4):255-259.

6. Agarwal N, Kriplani A, Parul GN, Hemal AK, Karak AK. Intramural bladder endometriosis after cesarean section: diagnostic and therapeutic aspects. J Gynecol Surg 2002 Jun;18(2):69-73.

7. Gupta S, Shah S, Motashaw ND, Shah N, Darshana V, Dave V. Case report: bladder wall endometrioma. Indian J Radiol Imaging 2001 Jan-Mar;11(1):23-24.

8. Abu J, Wong MYC, Foo KT, Yu SL. A case report on vesicouterine fistula: a very rare complication of the lower caesarean section. Singapore Med J 2000 Nov;41(11):554-556.

9. Shanmugasundaram R, Gopalakrishnan G, Kekre NS. Youssef's syndrome: is there a better way to diagnose? Indian J Urol 2008 Apr-Jun;24(2):269-270. 
